\title{
Jugendstrafvollzugsgesetz: Ein Schritt nach vorn oder zurück in die Kleinstaaterei?
}

Joachim Walter

U nter dem 28.04.2004 hat das Bundesministerium der Justiz den Bundesländern und den Fachverbänden einen neuen Entwurf eines Jugendstrafvollzugsgesetzes zugeleitet. Damit erhält die Hoffnung wieder Nahrung, dass der Jugendstrafvollzug aus seinem gesetzlosen, wie viele meinen sogar verfassungswidrigen Zustand (vgl. die Beiträge in Pollähne/Bammann/Feest 2004; zuletzt Butz, 2004) endlich erlöst werden könnte.

\section{Anlehnungsbedürfnis}

Ein erster Blick in den Entwurf zeigt, dass er sich in Aufbau und Inhalt weitgehend an dem immerhin gut bewährten - Strafvollzugsgesetz für Erwachsene von 1977 orientiert. Dies muss nicht dazu führen, dass die besonderen Bedürfnisse und Lebenslagen der Jugendlichen und Heranwachsenden übersehen werden. Vielmehr wird durchaus deutlich, dass es sich bei den Jugendstrafgefangenen nicht einfach um Häftlinge handelt, die eben etwas jünger sind. Es wird sehr wohl berücksichtigt, dass die jungen Menschen mitten in der Entwicklung stehen, deshalb gefördert werden können und müssen und nicht als Defizitwesen behandelt werden sollten. Die weitgehende Anlehnung an das Strafvollzugsgesetz birgt freilich die Gefahr, dass die Orientierung an der Entwicklungsfähigkeit junger Menschen nicht immer durchgehalten wird. So wird nicht durchgängig beachtet, dass es sich beim Jugendstrafvollzug im Verhältnis zum Erwachsenenvollzug sowohl sachlich als auch rechtlich um etwas qualitativ anderes, um ein aliud handelt. Aber immerhin würde die Bundesrepublik mit Erlass dieses Gesetzes - und das ist ja schon etwas - ihre Verpflichtung weit besser als zuvor erfüllen, die sie durch Ratifizierung internationaler Abkommen, insbesondere der UN-Kinderrechtskonvention, eingegangen ist, nämlich jugendgemäße Rechtsvorschriften für den Freiheitsentzug bei Jugendlichen zu erlassen.

\section{Grundsätze}

In seiner Grundsatzvorschrift (§ 2) legt der Entwurf fest, Ziel des Jugendstrafvollzuges sei »eine Lebensführung der Gefangenen ohne Straftaten«. In einem zukünftige Legalbewährung anstrebenden Jugendstrafvollzug geht es notwendig um Lern- und
Entwicklungsprozesse, auf die gehofft wird und die zu fördern sind. Und in der Tat ist Förderung einer der zentralen Begriffe des Entwurfs. Damit wird die Formulierung des § 1 SGB VIII aufgenommen, der jedem jungen Menschen, und sei er auch in Haft, »ein Recht auf Förderung seiner Entwicklung und auf Erziehung zu einer eigenverantwortlichen und gemeinschaftsfähigen Persönlichkeit« gewährt. Auch wenn in der Begründung (S. 14) ausdrücklich betont wird, dass damit keineswegs das Leitprinzip Erziehung verabschiedet werden soll, erscheint die nun gewählte Begrifflichkeit nüchterner, bescheidener und auch weniger ideologisch aufgeladen als die altväterliche Formulierung (»rechtschaffenen und verantwortungsbewussten Lebenswandel führen «) in § 91 JGG. Anlass der Inhaftierung sind ja Straftaten und nicht, wie es allerdings der Begriff der »schädlichen Neigungen « (§ 17 Abs. 2 JGG) nahe legt, Erziehungsdefizite. Überzogene Erwartungen an die Wirksamkeit des heute im Durchschnitt etwa ein Jahr dauernden Aufenthalts im Jugendstrafvollzug dürften so besser vermieden werden, als wenn man weiterhin das Banner der »Gesamterziehung « vor sich her trägt. § 9 Abs. 2 und $\S 10$ gewähren sogar einen Rechtsanspruch auf Erstellung eines Förderplans sowie auf Beteiligung des Gefangenen bei dessen Erarbeitung. Einvernehmliche Fördervereinbarungen werden angestrebt. Statt der in vielen Konzepten des Jugendstrafvollzugs bis heute dominierenden Defizitorientierung zeigt sich hier eine primäre Orientierung an der Entwicklungsfähigkeit junger Menschen, die in einen Rechtsanspruch für alle Gefangenen auf Förderung in der Entwicklung während des Jugendstrafvollzuges mündet.

Was gibt es sonst Positives zu vermelden? In erster Linie, dass die Eingriffe in Grundrechte in aller Regel hinreichend genau normiert sind, so dass dem Vorbehalt des Gesetzes und dem Bestimmtheitsgrundsatz Genüge getan ist. Der aus diesem Grund oft genug erhobene Vorwurf der Verfassungswidrigkeit dürfte damit erledigt sein. Gendermainstreaming ist wenigstens als Grundsatz im Gesetz verankert ( $\$ 5$ Abs. 2). Angleichungs-, Gegensteuerungs- und Eingliederungsgrundsatz sind aufgenommen; einige wenige gesetzliche Mindeststandards bezüglich Anstaltsgröße, Überbelegung, Unterbringung und Personalausstattung sind festgeschrieben. Die wissenschaftliche Evaluation des Jugendvollzugs und seiner Programme ist als Sollvorschrift ebenso vorgesehen wie Fortbildung und Supervision der Bediensteten.

\section{Irrweg und Normenfalle}

Der gravierendste Mangel des Entwurfs ist darin zu sehen, dass nach $\S 4$ die Gefangenen »verpflichtet (sind), an der Erreichung des Vollzugsziels mitzuwirken «. Damit wird die entsprechende Vorschrift des Strafvollzugsgesetzes für Erwachsene, dort § 4 Abs. 1, geradezu auf den Kopf gestellt, indem aus einem Recht zur Mitwirkung an der Gestaltung des Vollzugs eine generelle Mitwirkungspflicht gemacht wurde. Dies erscheint aus verfassungsrechtlichen, kriminologischen, pädagogischen und vollzugspraktischen Gründen unhaltbar. Zunächst einmal kann niemand von Rechts wegen, nämlich aus Anlass von Straftaten und durch Strafurteil, zur (aktiven) Mitwirkung an seiner eigenen »(Um-)Erziehung « verpflichtet werden. Allenfalls muss er diese (passiv) dulden. Unstreitig beinhaltet $\S 4 \mathrm{Ab}$ satz 1 StVollzG gerade keine Mitwirkungspflicht, weil der Gefangene nach Meinung des Gesetzgebers von 1976 im Hinblick auf seine Menschenwürde und Selbstverantwortung nicht zum bloßen Objekt behördlicher Bemühungen gemacht werden darf. Wie will man begründen (der Entwurf unterlässt es), dass Jugendstrafgefangene in dieser Hinsicht anders behandelt werden? Damit, dass sie sich noch in der Entwicklung befinden? Dann würde bei einem Eingriff in Freiheitsrechte negativ nach dem altersmäßigen Entwicklungsstand eines Menschen differenziert, der doch allenfalls geeignet ist, ihn zu exkulpieren. Vom Verstoß gegen das Verbot der Schlechterstellung Jugendlicher gegenüber Erwachsenen in vergleichbarer rechtlicher Lage ganz zu schweigen.

Anstatt den Jugendlichen von vornherein eine Rechtspflicht zur Mitwirkung aufzuerlegen (und auf die faktische Kraft des Normativen zu setzen), wird es vielmehr darauf ankommen, auf die Gefangenen mit individualisierten Angeboten zuzugehen und zu versuchen, sie aus der - auf dem Hintergrund ihrer bisherigen Biographie oft nur zu verständlichen - Ablehnung und Unwilligkeit heraus zur Mitarbeit zu motivieren. Aus vollzugspraktischer Sicht ist anzumerken, dass es ohnehin nicht möglich ist, jemanden zum Lernen zu zwingen, weil es eben sinnlos ist, »den Hund zum Jagen $\mathrm{zu}$ tragen «. Aus erziehungswissenschaftlicher Sicht ist hinzuzufügen, dass hier eine völlig antiquierte Auffassung sozio-moralischen Lernens zu Tage tritt, die statt auf Partizipation und Aushandlungsprozesse auf Paternalismus, auf unilaterale Kommunikation setzt. 
Durch die konstituierte allgemeine Mitwirkungspflicht wird, schlimm genug, nicht nur eine klassische Normenfalle aufgebaut, indem die oft genug missbrauchte Blankettformel »mangelnde Mitarbeit an der Erreichung des Vollzugszieles«, die jedoch bisher meist nur für die Versagung von Vollzugslockerungen herhalten musste, nunmehr als Universalbegründung für jedwede negative Entscheidung zu Lasten des Gefangenen gebraucht werden kann. Sondern es könnte dadurch die im Jugendstrafvollzug im Vergleich zum Erwachsenenvollzug ohnehin deutlich überhöhte Zahl der Disziplinarmaßnahmen (Dünkel 1996, S. 128) noch gesteigert werden: Gleichgültig aus welchem Grund der Gefangene seine Mitwirkung verweigert, immer wäre dies als Verletzung einer gesetzlichen Pflicht interpretierbar - und disziplinierbar (so auch Eisenberg, 2004 S. 355). Fast noch schlimmer ist, dass die Pflicht der Gefangenen zur Mitwirkung an der Erreichung des Vollzugsziels von der Verantwortlichkeit des Jugendvollzugs für die ihm zeitweise anvertrauten jungen Menschen ablenkt und versucht, die Verantwortung für gescheiterte Vollzugsverläufe von vornherein auf diese abzuwälzen. Geht's schief, liegt's im Zweifel nicht an der Institution, sondern an mangelnder Mitwirkung des Gefangenen. So wird letztlich auch der gerade eben zugestandene gesetzliche Anspruch der jungen Gefangenen auf Förderung in ihrer Entwicklung wieder relativiert bzw. negiert.

\section{Kostenfurcht und Zaghaftigkeit}

Aus dem Schicksal früherer Entwürfe lernend, die allesamt am Einspruch der Länderfinanzminister gescheitert sind, bemüht sich der Entwurf von vornherein, kostenträchtige Regelungen zu vermeiden. Deshalb verwundert es kaum, dass zahlreiche in der Fachliteratur aufgestellte Forderungen wie z. B. diejenigen nach einer stärkeren Belohnungsorientierung des Jugendstrafvollzuges, der Einführung gesetzlicher Mindeststandards bezüglich der Schulausbildung, der Berufsausbildung, des Sports und des Freizeitwesens oder Schlüsselzahlen für die Personalausstattung unbeachtet geblieben sind.

Weniger verständlich ist allerdings, dass auch zahlreiche Vorschläge, die nichts gekostet hätten, unter den Tisch gefallen sind, wie

- die Abschaffung der isolierenden Einzelhaft, die in den mit Zustimmung der Bundesrepublik verabschiedeten »Regeln der Vereinten Nationen zum Schutz von Jugendlichen unter Freiheitsentzug « vom 14.12.1990 in ihrer Nr. 66 ausdrücklich empfohlen wird;

- die Übernahme der Nr. 65 aus denselben UNRegeln, wonach in freiheitsentziehenden Einrichtungen für Jugendliche der Gebrauch und das Tragen von Waffen zu verbieten ist;
- ein Diskriminierungsverbot betreffend die Angehörigen von Minoritäten, das der Europarat in seiner Empfehlung Nr. R (88) 6 fordert,

- die Verankerung des Prinzips der Gewaltlosigkeit der Erziehung im Gesetz,

- der Vorrang offener Vollzugsformen und die Verpflichtung der Anstalten, offene Einrichtungen zu betreiben,

- der Vorrang der Erziehung vor Disziplinierung und daraus folgend ein gesetzlich definierter Ermessensspielraum bezüglich der Erstattung von Strafanzeigen,

um nur einige Beispiele zu nennen (ausführlich Walter 2004)

\section{Trotz alledem}

Obgleich mit mancherlei Mängeln behaftet, ermöglicht der Entwurf eine sachliche Diskussion, die aber wegen der notorischen Zweifel an der Verfassungsmäßigkeit des Jugendstrafvollzuges (umfassend Butz, 2004) auf das Ziel einer baldigen Verabschiedung eines Jugendstrafvollzugsgesetzes ausgerichtet sein sollte. Dazu passt es allerdings überhaupt nicht, dass nach einem Vorschlag der Bundesregierung in der Föderalismuskommission die Gesetzgebungskompetenz für den Strafvollzug und damit auch der Jugendstrafvollzug den Ländern übertragen werden soll. Wie kann man sich eine solche Offerte erklären, obwohl das Bundesjustizministerium gerade erst den hier besprochenen Entwurf für ein Jugendstrafvollzugsgesetz und neuerdings (22.09.2004) einen weiteren Entwurf für ein Gesetz zur Regelung des Vollzugs der Untersuchungshaft vorgelegt hat? Hat man sich gedacht, dass mit (Jugend) Strafvollzug ohnehin keine Wählerstimmen zu gewinnen sind? Will man Auseinandersetzungen mit den Bundesländern aus dem Weg gehen und ein leidiges Thema los werden?

Klar ist jedenfalls, dass die knapp 7000 in deutschen Jugendgefängnissen einsitzenden jungen Menschen hierzulande so gut wie keine Lobby haben. Obgleich es sich ein enorm überaltertes Land wie die Bundesrepublik Deutschland eigentlich nicht leisten kann, auch nur einen einzigen dieser jungen Menschen verloren zu geben.

Was sind die zu erwartenden Folgen einer solchen Kompetenzverlagerung, ja schon des Vorschlags einer solchen? Zunächst einmal die, dass zumindest einige Länder ihre Zustimmung zum jetzt vorliegenden Entwurf solange hinausziehen werden, bis sie selbst die Gesetzgebungskompetenz haben, um danach ein für das eigene Land (oder Ländchen) "maßgeschneidertes « Jugendstrafvollzugsgesetz ohne störende Kompromisserfordernisse zu basteln. Ob daraus allerdings auch Maßanzüge für die betroffenen Jugendlichen werden, ist zu bezweifeln, zumal das materielle Strafrecht, das Strafprozessrecht und das Strafvollstreckungsrecht in der Bundeskompetenz bleiben werden. Vielmehr würde eine solche Kompetenzverlagerung das Einfallstor für Augenblicksstimmungen oder gar Sicherheitspaniken in das Vollzugsrecht darstellen und symbolische Gesetzgebungsaktionen der Länder begünstigen. Das Ergebnis wäre, dass statt bundeseinheitlicher Rechtsgarantien und Qualitätsstandards ein vollzuglicher Fleckerlteppich entsteht, der die länderübergreifende $\mathrm{Zu}$ sammenarbeit ebenso erschwert wie er die Arbeitsmöglichkeiten der Straffälligenhilfe behindert.

Weiterhin ist zu befürchten, dass zwischen den Ländern, gerade auch in Wahlkampfzeiten, ein Wettlauf um den einerseits billigsten und andererseits »härtesten" Jugendstrafvollzug entstehen könnte. Nicht auszudenken der Schaden, wenn irgendwo in einem der 16 Bundesländer ein populistischer Politiker vom Schlage des Richters Schill Justizminister würde und es ihm gelänge, seine punitiven Vorstellungen aus dem vorvergangenen Jahrhundert Gesetz werden zu lassen. Gewiss, da ist noch das Bundesverfassungsgericht! Nur: Jugendstrafgefangene pflegen äußerst selten den Rechtsweg zu beschreiten, und wenn doch, dann müssten sie diesen zunächst erschöpft haben, um mit ihrer Beschwerde beim Bundesverfassungsgericht anzukommen. Das dürfte dauern ... Fazit: Dem Jugendstrafvollzug und dem Strafvollzug als ganzem kann nichts Schlimmeres passieren, als dass der Bund die Gesetzgebungskompetenz auf die Länder überträgt. Ein Rückfall in die Zeit der Duodezfürstentümer wäre vorprogrammiert.

Was also ist zu tun? Die Rechtspolitiker sollten sich aufraffen und ungeachtet mancher berechtigter Meinungsunterschiede auf der Grundlage des vorliegenden Entwurfes ein Jugendstrafvollzugsgesetz sobald wie möglich verabschieden, in jedem Fall noch in der laufenden Legislaturperiode. Einen Rückfall in die kriminalpolitische Kleinstaaterei können wir uns ebenso wenig leisten wie eine Fortsetzung des gesetzlosen Zustandes.

\section{Literatur:}

Butz, Katharina (2004): die Verhängung von Jugendstrafe vor dem Hintergrund der Verfassungswidrigkeit des Jugendstrafvollzuges. Untersuchung zur Verfassungsgemäßheit von $\S 17$ Abs. 2 JGG. Shaker Verlag, Aachen.

Dünkel, Frieder (1996): Empirische Forschung im Strafvollzug. Bestandsaufnahme und Perspektiven. Forum Verlag, Bonn/Mönchengladbach.

Eisenberg, Ulrich (2004): Zum RefE eines JStVollzG des BMJ vom 28. 4. 2004. Monatsschrift für Kriminologie und Strafrechtsreform 2004, S. 353.

Pollähne, Helmut / Bammann, Kai / Feest, Johannes (Hrsg.) (2004): Wege aus der Gesetzlosigkeit. Rechtslage und Regelungsbedürftigkeit des Jugendstrafvollzugs. Schriftenreihe der deutschen Vereinigung für Jugendgerichte und Jugendgerichtshilfen e.V. Forum Verlag Godesberg, Mönchengladbach.

Walter, Joachim (2004): Der neue Entwurf eines Gesetzes zur Regelung des Jugendstrafvollzuges aus praktischer Sicht. Zentralblatt für Jugendrecht 2004, S. $397-405$.

Dr. Joachim Walter ist Leiter der Justizvollzugsanstalt Adelsheim und Mitherausgeber dieser Zeitschrift 